\title{
Novel hyperbolic and exponential ansatz methods to the fractional fifth-order Korteweg-de Vries equations
}

\author{
Choonkil Park ${ }^{1 *}$, R.I. Nuruddeen ${ }^{2}$, Khalid K. Ali ${ }^{3}$, Lawal Muhammad ${ }^{4}$, M.S. Osmann ${ }^{5,6^{*}}$ (iD and \\ Dumitru Baleanu ${ }^{7,8^{*}}$
}

"Correspondence:

baak@hanyang.ac.kr;

mofatzi@sci.cu.edu.eg;

dumitru@cankaya.edu.tr

${ }^{1}$ Research Institute for Natural

Sciences, Hanyang University, Seoul

04763, South Korea

${ }^{5}$ Department of Mathematics,

Faculty of Science, Cairo University,

Giza 12613, Egypt

Full list of author information is

available at the end of the article

\begin{abstract}
This paper aims to investigate the class of fifth-order Korteweg-de Vries equations by devising suitable novel hyperbolic and exponential ansatze. The class under consideration is endowed with a time-fractional order derivative defined in the conformable fractional derivative sense. We realize various solitons and solutions of these equations. The fractional behavior of the solutions is studied comprehensively by using $2 \mathrm{D}$ and $3 \mathrm{D}$ graphs. The results demonstrate that the methods mentioned here are more effective in solving problems in mathematical physics and other branches of science.
\end{abstract}

Keywords: Fractional derivative; Fifth-order KdV equations; Hyperbolic wave solutions; Exponential wave solutions; Solitary wave solutions

\section{Introduction}

Nonlinear partial differential equations (PDEs) play a significant role in several scientific and engineering fields [1-5]. Since the discovery of the soliton in 1965 by Zabusky and Kruskal [6], many nonlinear PDEs have been derived and extensively applied in different branches of physics and applied mathematics [7-17]. Nonlinear PDEs appear in condensed matter, solid state physics, fluid mechanics, chemical kinetics, plasma physics, nonlinear optics, propagation of fluxion in Josephson junctions, ocean dynamics and many others [18-27]. In order to understand the different nonlinear phenomena, various methods for obtaining exact solutions to nonlinear PDEs have been proposed [28-31].

One of the most interesting evolution equations with a lot of applications in describing different phenomena is the Korteweg-de Vries equation [32, 33]. This equation occurs in different types, orders and lots of modifications [34-46]. Certain applications of the equation are found in many fields including fluids dynamics, plasma physics and shallow water and nonlinear waves processes, respectively. The main motivation of this work is to study the fifth-order Korteweg-de Vries equation endowed with a time-fractional order derivative in time that reads [47]

$$
u_{t}^{\alpha}+a u^{2} u_{x}+b u_{x} u_{x x}+c u u_{x x x}+d u_{x x x x x}=0
$$

(c) The Author(s) 2020. This article is licensed under a Creative Commons Attribution 4.0 International License, which permits use sharing, adaptation, distribution and reproduction in any medium or format, as long as you give appropriate credit to the original author(s) and the source, provide a link to the Creative Commons licence, and indicate if changes were made. The images or other third party material in this article are included in the article's Creative Commons licence, unless indicated otherwise in a credit line to the material. If material is not included in the article's Creative Commons licence and your intended use is not permitted by statutory regulation or exceeds the permitted use, you will need to obtain permission directly from the copyright holder. To view a copy of this licence, visit http://creativecommons.org/licenses/by/4.0/. 
where $\alpha \in(0,1]$ is the fractional order derivative and $a, b, c$ and $d$ are non-zero real constants. The fractional order derivative $\alpha$ in the above equation is considered to be taken in the recent conformable fractional derivative sense [48-50]. It is worth to be noticed that the field of fractional calculus is an old area of research that has gained much interest in the last few decades [51-56].

Many researchers have proposed various forms of Eq. (1) by suitably introducing different values of the non-zero real constants $a, b, c$ and $d$. Some of the famous examples with fractional order derivatives in time include [47]:

1) The fractional Sawada-Kotera equation

$$
u_{t}^{\alpha}+45 u^{2} u_{x}+15 u_{x} u_{x x}+15 u u_{x x x}+u_{x x x x x}=0
$$

2) the fractional Caudrey-Dodd-Gibbon equation

$$
u_{t}^{\alpha}+180 u^{2} u_{x}+30 u_{x} u_{x x}+30 u u_{x x x}+u_{x x x x x}=0,
$$

3) the fractional Lax equation

$$
u_{t}^{\alpha}+30 u^{2} u_{x}+30 u_{x} u_{x x}+10 u u_{x x x}+u_{x x x x x}=0
$$

4) the fractional Kaup-Kuperschmidt equation

$$
u_{t}^{\alpha}+20 u^{2} u_{x}+25 u_{x} u_{x x}+10 u u_{x x x}+u_{x x x x x}=0
$$

and

5) the fractional Ito equation

$$
u_{t}^{\alpha}+2 u^{2} u_{x}+6 u_{x} u_{x x}+3 u u_{x x x}+u_{x x x x x}=0 .
$$

However, we tackle in this paper the class of time-conformable fractional fifth-order Korteweg-de Vries equations given in Eqs. (2)-(6) by devising suitable novel hyperbolic and exponential ansatze. The essential advantage of these techniques over the other methods in the literature is that they present novel explicit analytical wave solutions including many real free parameters. The closed-form wave answers of nonlinear PDEs have a significant meaning revealing the interior working of the physical phenomena. Furthermore, the calculations in these methods are very simple and vital in presenting new solutions compared with the steps in other approaches.

In doing so, various solitons and solutions of the equations will be realized and further depicted graphically to confirm their shapes. One can well see various analytical and numerical methods used in tackling different forms of the Korteweg-de Vries equations and evolution equations in the papers cited above and also in [57-61]. The current paper is organized as follows: Sect. 2 presents some preliminaries about the conformable fractional derivative. Section 3 gives the concept of the methodology. Section 4 is reserved for the application. Section 5 gives some graphical results and a discussion. Finally, Sect. 6 gives the conclusion. 


\section{Fractional conformable derivative}

Definition 1 ([48]) Let $u:[0, \infty) \rightarrow \mathbb{R}$ be a real-valued function. The fractional conformable derivative of order $\alpha$ for $u(t)$ is defined by

$$
D_{t}^{\alpha}(u(t))=\lim _{\delta \rightarrow 0}\left(\frac{u\left(t+\delta t^{1-\alpha}\right)-u(t)}{\delta}\right), \quad t>0, \alpha \in(0,1] .
$$

Theorem 1 ([48]) Suppose $v(t)$ and $w(t)$ are $\alpha$-differentiable for $\alpha \in(0,1]$ and $t>0$, then

(a) $D_{t}^{\alpha}\left(t^{n}\right)=n t^{n-\alpha}, \forall n \in \mathbb{R}$,

(b) $D_{t}^{\alpha}(C)=0, \forall C \in \mathbb{R}$

(c) $D_{t}^{\alpha}(x v(t)+y w(t))=x D_{t}^{\alpha} v(t)+y D_{t}^{\alpha} w(t), \forall x, y \in \mathbb{R}$,

(d) $D_{t}^{\alpha}(v(t) w(t))=v(t) D_{t}^{\alpha}(w(t))+w(t) D_{t}^{\alpha}(v(t))$,

(e) $D_{t}^{\alpha}\left(\frac{v(t)}{w(t)}\right)=\frac{1}{w^{2}(t)}\left(w(t) D_{t}^{\alpha} v(t)-v(t) D_{t}^{\alpha} w(t)\right), w(t) \neq 0$,

(f) Importantly, if the first derivative of $v(t)$ exists, then

$$
D_{t}^{\alpha} v(t)=t^{1-\alpha} \frac{d v}{d t}
$$

Theorem 2 ([48]) Let it be given that $v(t)$ is $\alpha$-differentiable for $\alpha \in(0,1]$. Let $w(t)$ be a differentiable function defined in the range of $v(t)$, then

$$
D_{t}^{\alpha}(v(t) \circ w(t))=t^{1-\alpha} w^{\prime}(t) v^{\prime}(w(t)) .
$$

The proof of Theorem 1 and Theorem 2 can be found in [48].

\section{The hyperbolic and exponential ansatz methods}

We consider the nonlinear time-fractional differential equation of the form

$$
P\left(u, D_{t}^{\alpha} u, D_{x} u, D_{t t}^{2 \alpha} u, D_{x x} u, D_{t}^{\alpha} D_{x} u, \ldots\right)=0,
$$

where $\alpha \in(0,1]$ is the fractional order derivative. Further, we make use of the following transformations:

$$
u(x, t)=v(\xi), \quad \xi=f\left(x, \frac{t^{\alpha}}{\alpha}\right)
$$

where $f$ is a function of $x$ and $t$, depending on the application. Moreover, we derive the following hyperbolic (see periodic ansatze in [35]) and exponential [41] ansatze for transformations for the fractional fifth-order Korteweg-de Vries equation under consideration.

1) Hyperbolic ansatz method

$$
v(\xi)= \begin{cases}A_{0}+A_{1} \operatorname{sech}^{2}(\xi), & \text { bright soliton solution, } \\ A_{0}+A_{1} \tanh ^{2}(\xi), & \text { dark soliton solution, } \\ A_{0}+A_{1} \operatorname{csch}^{2}(\xi), & \text { singular soliton solution, } \\ A_{0}+A_{1} \operatorname{coth}^{2}(\xi), & \text { singular soliton solution. }\end{cases}
$$


2) Exponential ansatz method

$$
v(\xi)=A_{1}+A_{2} \frac{e^{\xi}}{\left(1+e^{\xi}\right)^{2}},
$$

where $A_{0}, A_{1} \neq 0$ and $A_{2} \neq 0$ are non-zero constants to be determined. Substituting either Eq. (12) or (13) as the case maybe into Eq. (10) and retaining the relevant coefficients in $\xi$, we get a system of algebraic equations. This system of equations will then be solved simultaneously to determine the unknowns with the help of computer software to obtain the solutions of Eq. (10).

\section{Application}

In this section, we employ the presented hyperbolic and exponential ansatz function methods to construct bright soliton solutions, dark soliton solutions, singular soliton solutions and exponential solutions for the class of fractional fifth-order Korteweg-de Vries equations under consideration.

\subsection{The fractional Sawada-Kotera equation}

\subsubsection{Bright soliton solution}

Let $A_{0}, A_{1} \neq 0, k, s$ and $w$ be arbitrary constants. Then we assume a bright soliton solution of the form

$$
u(x, t)=A_{0}+A_{1} \operatorname{sech}^{2}(\xi), \quad \xi=s x-k \frac{t^{\alpha}}{\alpha}+w .
$$

Substituting Eq. (14) into (2) and simplify as explained in Sect. 3, we get the following system of algebraic equations:

$$
\begin{aligned}
& -2 A_{1} k+32 A_{1} s^{5}+120 A_{0} A_{1} s^{3}+90 A_{0}^{2} A_{1} s=0, \\
& -480 A_{1} s^{5}+240 A_{1}^{2} s^{3}-360 A_{0} A_{1} s^{3}+180 A_{0} A_{1}^{2} s=0, \\
& 720 A_{1} s^{5}-540 A_{1}^{2} s^{3}+90 A_{1}^{3} s=0 .
\end{aligned}
$$

Solving the above system gives

$$
A_{0}=\frac{ \pm \sqrt{5} \sqrt{k s+4 s^{6}}-10 s^{3}}{15 s}, \quad A_{1}=2 s^{2}
$$

which yields the following bright soliton solution:

$$
u_{1}(x, t)=\frac{ \pm \sqrt{5} \sqrt{k s+4 s^{6}}-10 s^{3}}{15 s}+2 s^{2} \operatorname{sech}^{2}\left(s x-k \frac{t^{\alpha}}{\alpha}+w\right) .
$$

\subsubsection{Dark soliton solution}

Let $A_{0}, A_{1} \neq 0, k, s$ and $w$ be arbitrary constants. Then we assume a bright soliton solution of the form

$$
u(x, t)=A_{0}+A_{1} \tanh ^{2}(\xi), \quad \xi=s x-k \frac{t^{\alpha}}{\alpha}+w .
$$


Inserting Eq. (18) into (2), we get the following system of algebraic equations:

$$
\begin{aligned}
& 2 A_{1} k-272 A_{1} s^{5}-60 A_{1}^{2} s^{3}+240 A_{0} A_{1} s^{3}-90 A_{0}^{2} A_{1} s=0, \\
& -2 A_{1} k+1232 A_{1} s^{5}+540 A_{1}^{2} s^{3}-600 A_{0} A_{1} s^{3}-180 A_{0} A_{1}^{2} s+90 A_{0}^{2} A_{1} s=0, \\
& -1680 A_{1} s^{5}-1020 A_{1}^{2} s^{3}+360 A_{0} A_{1} s^{3}-90 A_{1}^{3} s+180 A_{0} A_{1}^{2} s=0, \\
& 720 A_{1} s^{5}+540 A_{1}^{2} s^{3}+90 A_{1}^{3} s=0 .
\end{aligned}
$$

Solving the above system gives

$$
A_{0}=\frac{20 s^{3} \pm \sqrt{5} \sqrt{k s+4 s^{6}}}{15 s}, \quad A_{1}=-2 s^{2},
$$

which yields the following dark soliton solution:

$$
u_{2}(x, t)=\frac{20 s^{3} \pm \sqrt{5} \sqrt{k s+4 s^{6}}}{15 s}-2 s^{2} \tanh ^{2}\left(s x-k \frac{t^{\alpha}}{\alpha}+w\right) .
$$

\subsection{The fractional Caudrey-Dodd-Gibbon equation}

\subsubsection{Singular soliton solutions}

Let $A_{0}, A_{1} \neq 0, s, k$ and $w$ be arbitrary constants. We have the following two cases:

Case (I). Assume a singular soliton solution of the form

$$
u(x, t)=A_{0}+A_{1} \operatorname{csch}^{2}(\xi), \quad \xi=s x-k \frac{t^{\alpha}}{\alpha}+w .
$$

Inserting Eq. (22) into (3), we get the following system of algebraic equations:

$$
\begin{aligned}
& A_{1} k-32 A_{1} s^{5}-240 A_{0} A_{1} s^{3}-360 A_{0}^{2} A_{1} s=0, \\
& -480 A_{1} s^{5}-480 A_{1}^{2} s^{3}-720 A_{0} A_{1} s^{3}-720 A_{0} A_{1}^{2} s=0, \\
& -720 A_{1} s^{5}-1080 A_{1}^{2} s^{3}-360 A_{1}^{3} s=0 .
\end{aligned}
$$

Thus, solving the above system gives

$$
A_{0}=\frac{ \pm \sqrt{5} \sqrt{k s+4 s^{6}}-10 s^{3}}{30 s}, \quad A_{1}=-s^{2},
$$

which yields the following singular soliton solution:

$$
u_{3}(x, t)=\frac{ \pm \sqrt{5} \sqrt{k s+4 s^{6}}-10 s^{3}}{30 s}-s^{2} \operatorname{Csch}^{2}\left(s x-k \frac{t^{\alpha}}{\alpha}+w\right) .
$$

Case (II). Assume a singular soliton solution of the form

$$
u(x, t)=A_{0}+A_{1} \operatorname{coth}^{2}(\xi), \quad \xi=s x-k \frac{t^{\alpha}}{\alpha}+w .
$$

Inserting Eq. (26) into (3), we get the following system of algebraic equations:

$$
2 A_{1} k-272 A_{1} s^{5}-120 A_{1}^{2} s^{3}+480 A_{0} A_{1} s^{3}-360 A_{0}^{2} A_{1} s=0,
$$




$$
\begin{aligned}
& -2 A_{1} k+1232 A_{1} s^{5}+1080 A_{1}^{2} s^{3}-1200 A_{0} A_{1} s^{3}-720 A_{0} A_{1}^{2} s+360 A_{0}^{2} A_{1} s=0, \\
& -1680 A_{1} s^{5}-2040 A_{1}^{2} s^{3}+720 A_{0} A_{1} s^{3}-360 A_{1}^{3} s+720 A_{0} A_{1}^{2} s=0, \\
& 720 A_{1} s^{5}+1080 A_{1}^{2} s^{3}+360 A_{1}^{3} s=0 .
\end{aligned}
$$

Thus, solving the above system gives

$$
A_{0}=\frac{20 s^{3} \pm \sqrt{5} \sqrt{k s+4 s^{6}}}{30 s}, \quad A_{1}=-s^{2}
$$

which yields the following singular soliton solution:

$$
u_{4}(x, t)=\frac{20 s^{3} \pm \sqrt{5} \sqrt{k s+4 s^{6}}}{30 s}-s^{2} \operatorname{coth}^{2}\left(s x-k \frac{t^{\alpha}}{\alpha}+w\right) .
$$

\subsection{The fractional Lax equation}

\subsubsection{Bright soliton solution}

Let $A_{0}, A_{1} \neq 0, s, k$ and $w$ be arbitrary constants. Assume a bright soliton solution of the form

$$
u(x, t)=A_{0}+A_{1} \operatorname{sech}^{2}(\xi), \quad \xi=s x-k \frac{t^{\alpha}}{\alpha}+w
$$

Substituting Eq. (30) into (4), we get the following system of algebraic equations:

$$
\begin{aligned}
& -2 A_{1} k+32 A_{1} s^{5}+80 A_{0} A_{1} s^{3}+60 A_{0}^{2} A_{1} s=0, \\
& -480 A_{1} s^{5}+320 A_{1}^{2} s^{3}-240 A_{0} A_{1} s^{3}+120 A_{0} A_{1}^{2} s=0, \\
& 720 A_{1} s^{5}-600 A_{1}^{2} s^{3}+60 A_{1}^{3} s=0 .
\end{aligned}
$$

Thus, solving the above system gives

$$
A_{0}=\frac{1}{3}\left( \pm \sqrt{13} s^{2}-5 s^{2}\right), \quad A_{1}=\mp \sqrt{13} s^{2}+5 s^{2}, \quad k=4\left(\mp 5 \sqrt{13} s^{5}+19 s^{5}\right)
$$

which yields the following bright soliton solution:

$$
u_{5}(x, t)=\frac{1}{3}\left( \pm \sqrt{13} s^{2}-5 s^{2}\right)+\left(\mp \sqrt{13} s^{2}+5 s^{2}\right) \operatorname{sech}^{2}\left(s x-k \frac{t^{\alpha}}{\alpha}+w\right) .
$$

\subsubsection{Dark soliton solution}

Let $A_{0}, A_{1} \neq 0, s, k$ and $w$ be arbitrary constants. Let us assume a bright soliton solution in the form

$$
u(x, t)=A_{0}+A_{1} \tanh ^{2}(\xi), \quad \xi=s x-k \frac{t^{\alpha}}{\alpha}+w
$$

Substituting Eq. (34) into (4), we get the following system of algebraic equations:

$$
\begin{aligned}
& 2 A_{1} k-272 A_{1} s^{5}-120 A_{1}^{2} s^{3}+160 A_{0} A_{1} s^{3}-60 A_{0}^{2} A_{1} s=0, \\
& -2 A_{1} k+1232 A_{1} s^{5}+760 A_{1}^{2} s^{3}-400 A_{0} A_{1} s^{3}-120 A_{0} A_{1}^{2} s+60 A_{0}^{2} A_{1} s=0,
\end{aligned}
$$




$$
\begin{aligned}
& -1680 A_{1} s^{5}-1240 A_{1}^{2} s^{3}+240 A_{0} A_{1} s^{3}-60 A_{1}^{3} s+120 A_{0} A_{1}^{2} s=0, \\
& 720 A_{1} s^{5}+600 A_{1}^{2} s^{3}+60 A_{1}^{3} s=0 .
\end{aligned}
$$

Thus, solving the above system gives

$$
A_{0}=\frac{2}{3}\left( \pm \sqrt{13} s^{2}+5 s^{2}\right), \quad A_{1}=-\sqrt{13} s^{2} \mp 5 s^{2}, \quad k=4\left(\mp 5 \sqrt{13} s^{5}+19 s^{5}\right)
$$

which yields the following dark soliton solution:

$$
u_{6}(x, t)=\frac{2}{3}\left( \pm \sqrt{13} s^{2}+5 s^{2}\right)+\left(\mp 5 \sqrt{13} s^{5}+19 s^{5}\right) \tanh ^{2}\left(s x-k \frac{t^{\alpha}}{\alpha}+w\right)
$$

\subsection{The fractional Kaup-Kuperschmidt equation}

\subsubsection{Exponential solution}

Let $A_{1}, A_{2} \neq 0, k$ and $c$ be arbitrary constants. We assume an exponential solution in the form

$$
u(x, t)=A_{1}+A_{2} \frac{e^{\xi}}{\left(1+e^{\xi}\right)^{2}}, \quad \xi=k\left(x-c \frac{t^{\alpha}}{\alpha}\right)
$$

Substituting Eq. (38) into (5), we get the following system of algebraic equations:

$$
\begin{aligned}
& 20 A_{1}^{2} A_{2} k+10 A_{1} A_{2} k^{3}-A_{2} c k+A_{2} k^{5}=0, \\
& 60 A_{1}^{2} A_{2} k+40 A_{1} A_{2}^{2} k-90 A_{1} A_{2} k^{3}+35 A_{2}^{2} k^{3}-3 A_{2} c k-57 A_{2} k^{5}=0, \\
& 40 A_{1}^{2} A_{2} k+40 A_{1} A_{2}^{2} k-100 A_{1} A_{2} k^{3}+20 A_{2}^{3} k-235 A_{2}^{2} k^{3}-2 A_{2} c k+302 A_{2} k^{5}=0, \\
& -40 A_{1}^{2} A_{2} k-40 A_{1} A_{2}^{2} k+100 A_{1} A_{2} k^{3}-20 A_{2}^{3} k+235 A_{2}^{2} k^{3}+2 A_{2} c k-302 A_{2} k^{5}=0, \\
& -60 A_{1}^{2} A_{2} k-40 A_{1} A_{2}^{2} k+90 A_{1} A_{2} k^{3}-35 A_{2}^{2} k^{3}+3 A_{2} c k+57 A_{2} k^{5}=0, \\
& -20 A_{1}^{2} A_{2} k-10 A_{1} A_{2} k^{3}+A_{2} c k-A_{2} k^{5}=0 .
\end{aligned}
$$

Thus, solving the above system gives

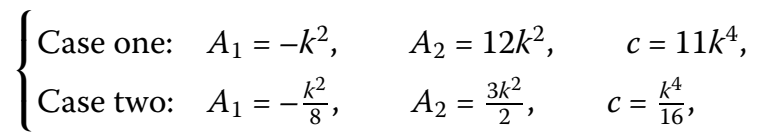

which yield the following solutions:

$$
u_{7}(x, t)=-k^{2}+\frac{12 k^{2} e^{k\left(x-11 k^{4} \frac{t^{\alpha}}{\alpha}\right)}}{\left(1+e^{k\left(x-11 k^{4} \frac{t^{\alpha}}{\alpha}\right)}\right)^{2}}
$$

or

$$
u_{8}(x, t)=-\frac{k^{2}}{8}+\frac{3 k^{2} e^{k\left(x-\frac{k^{4}}{16} \frac{t^{\alpha}}{\alpha}\right)}}{2\left(1+e^{k\left(x-\frac{k^{4}}{16} \frac{t^{\alpha}}{\alpha}\right)}\right)^{2}} .
$$




\subsection{The fractional Ito equation}

\subsubsection{Exponential solution}

Let $A_{1}, A_{2} \neq 0, k$ and $c$ be arbitrary constants. Assume we have an exponential solution in the form

$$
u(x, t)=A_{1}+A_{2} \frac{e^{\xi}}{\left(1+e^{\xi}\right)^{2}}, \quad \xi=k\left(x-c \frac{t^{\alpha}}{\alpha}\right) .
$$

Substituting Eq. (43) into (6), we get the following system of algebraic equations:

$$
\begin{aligned}
& 2 A_{1}^{2} A_{2} k-A_{2} c k+3 A_{1} A_{2} k^{3}+A_{2} k^{5}=0, \\
& 6 A_{1}^{2} A_{2} k+4 A_{1} A_{2}^{2} k-3 A_{2} c k-27 A_{1} A_{2} k^{3}+9 A_{2}^{2} k^{3}-57 A_{2} k^{5}=0, \\
& 4 A_{1}^{2} A_{2} k+4 A_{1} A_{2}^{2} k+2 A_{2}^{3} k-2 A_{2} c k-30 A_{1} A_{2} k^{3}-63 A_{2}^{2} k^{3}+302 A_{2} k^{5}=0, \\
& -4 A_{1}^{2} A_{2} k-4 A_{1} A_{2}^{2} k-2 A_{2}^{3} k+2 A_{2} c k+30 A_{1} A_{2} k^{3}+63 A_{2}^{2} k^{3}-302 A_{2} k^{5}=0, \\
& -6 A_{1}^{2} A_{2} k-4 A_{1} A_{2}^{2} k+3 A_{2} c k+27 A_{1} A_{2} k^{3}-9 A_{2}^{2} k^{3}+57 A_{2} k^{5}=0, \\
& -2 A_{1}^{2} A_{2} k+A_{2} c k-3 A_{1} A_{2} k^{3}-A_{2} k^{5}=0 .
\end{aligned}
$$

Thus, solving the above system gives

$$
A_{1}=-\frac{5 k^{2}}{2}, \quad A_{2}=30 k^{2}, \quad c=6 k^{4}
$$

which yield the following solutions:

$$
u_{9}(x, t)=-\frac{5 k^{2}}{2}+\frac{30 k^{2} e^{k\left(x-6 k^{4} \frac{t^{\alpha}}{\alpha}\right)}}{\left(1+e^{k\left(x-6 k^{4} \frac{t^{\alpha}}{\alpha}\right)}\right)^{2}} .
$$

\section{Graphical results and discussion}

In this section, we give some graphical depictions to some of the acquired solutions using the devised hyperbolic and exponential ansatz methods for the class of fifth-order Korteweg-de Vries equations under consideration. Both the 2-dimensional and the 3dimensional plots are presented. Based on the ansatz methods devised, we have constructed bright and dark soliton solutions for the fractional Sawada-Kotera equation in Eqs. (17) and (21); singular soliton solutions for the fractional Caudrey-Dodd-Gibbon in equations (25) and (29); bright and dark soliton solutions for the fractional Lax equation in Eqs. (33) and (37); exponential solutions for the fractional Kaup-Kuperschmidt in Eqs. (41) and (42); and finally the exponential solution for the fractional Ito in Eq. (46). In Fig. 1, we plot the dark soliton solution of the fractional Sawada-Kotera equation given in equation (19). In Fig. 2, we plot the singular solution of the fractional Caudrey-DoddGibbon given in Eq. (25). In Fig. 3, we plot the exponential solution of the fractional KaupKuperschmidt equation given in Eqs. (36). In these figures, we study the effect of the fraction order on the variation of the wave displacement. Figures 1 and 3 cleanly show bellshaped solution, while Fig. 2 gives a singular solution representation.

In Figs. 1-3, the fractional order $\alpha$ clearly affected on the propagating of wave solution in which the amplitude is increased with the increase of $\alpha$. 

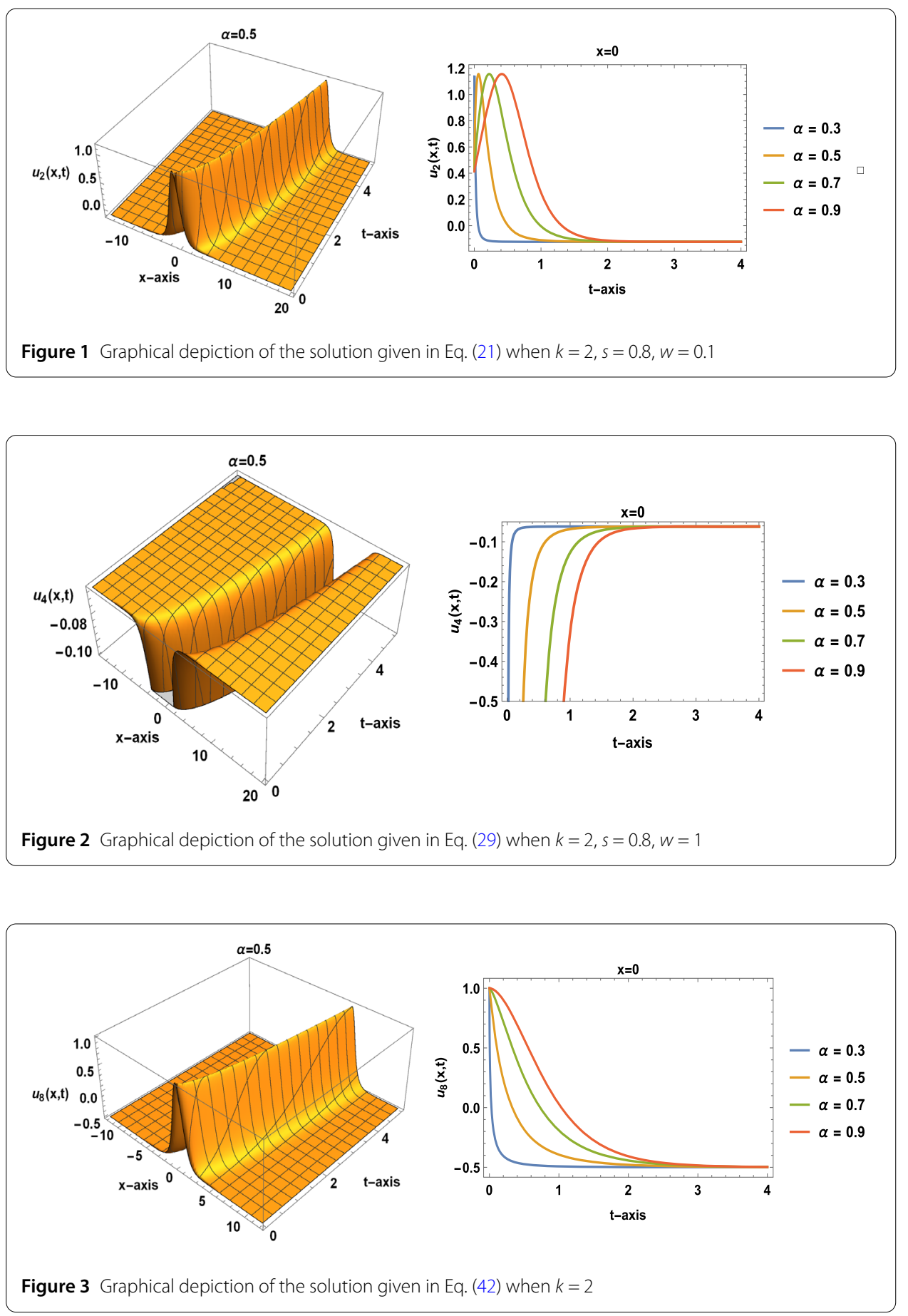

\section{Conclusion}

In summary, the present paper investigates a well-known class of fifth-order Korteweg-de Vries equations by devising novel hyperbolic and exponential ansatze in the presence of a time-fractional order derivative. The fractional derivative is considered to be taken in the sense of the conformable fractional derivative. Various solitons and solutions of the equations including bright solitons, dark solitons, singular solitons and certain exponential solutions have been realized in the study. We finally depict some of the obtained solutions 
graphically and conclude that similar considerations of various evolution equations can be done using the devised ansatze.

\author{
Acknowledgements \\ The authors are thankful to the editor and anonymous referees for their valuable comments and suggestions. \\ Funding \\ C. Park was supported by Basic Science Research Program through the National Research Foundation of Korea funded by \\ the Ministry of Education, Science and Technology (NRF-2017R1D1A1B04032937).
}

\title{
Availability of data and materials
}

Not applicable.

\section{Competing interests}

The authors declare that they have no competing interests.

\section{Authors' contributions}

All authors conceived of the study, participated in its design and coordination, drafted the manuscript, participated in the sequence alignment, and read and approved the final manuscript.

\section{Author details}

${ }^{1}$ Research Institute for Natural Sciences, Hanyang University, Seoul 04763, South Korea. ${ }^{2}$ Department of Mathematics, Faculty of Science, Federal University Dutse, Dutse, Jigawa State, Nigeria. ${ }^{3}$ Mathematics Department, Faculty of Science, Al-Azhar University, Nasr-City, Cairo, Egypt. ${ }^{4}$ Department of Mathematics, Faculty of Science, Yusuf Maitama Sule University, Kano, Kano State, Nigeria. ${ }^{5}$ Department of Mathematics, Faculty of Science, Cairo University, Giza 12613, Egypt. ${ }^{6}$ Department of Mathematics, Faculty of Applied Science, Umm Alqura University, Makkah 21955, Saudi Arabia. ${ }^{7}$ Department of Mathematics, Cankaya University, Ögretmenler Cad. 1406530, Ankara, Turkey. ${ }^{8}$ Institute of Space Sciences, Magurele, Bucharest, Romania.

\section{Publisher's Note}

Springer Nature remains neutral with regard to jurisdictional claims in published maps and institutional affiliations.

Received: 11 June 2020 Accepted: 27 October 2020 Published online: 07 November 2020

\section{References}

1. Kaur, D., Agarwal, P., Rakshit, M., Chand, M.: Fractional calculus involving $(p, q)$-Mathieu type series. Appl. Math. Nonlinear Sci. 5(2), 15-34 (2020)

2. Kumar, V.S., Rezazadeh, H., Eslami, M., Izadi, F., Osman, M.S.: Jacobi elliptic function expansion method for solving KdV equation with conformable derivative and dual-power law nonlinearity. Int. J. Appl. Comput. Math. 5(5), 127 (2019)

3. Agarwal, P., Attary, M., Maghasedi, M., Kumam, P.: Solving higher-order boundary and initial value problems via Chebyshev-spectral method: application in elastic foundation. Symmetry 12(6), 987 (2020)

4. Lu, D., Tariq, K.U., Osman, M.S., Baleanu, D., Younis, M., Khater, M.M.A.: New analytical wave structures for the $(3+1)$-dimensional Kadomtsev-Petviashvili and the generalized Boussinesq models and their applications. Results Phys. 14, 102491 (2019)

5. Lu, D., Osman, M.S., Khater, M.M.A., Attia, R.A.M., Baleanu, D.: Analytical and numerical simulations for the kinetics of phase separation in iron (Fe-Cr-X (X = Mo, Cu)) based on ternary alloys. Physica A 537, 122634 (2020)

6. Zabusky, N.J., Kruskal, M.D.: Interaction of "solitons" in a collisionless plasma and the recurrence of initial states. Phys. Rev. Lett. 15(6), 240 (1965)

7. Liu, J.G., Osman, M.S., Wazwaz, A.M.: A variety of nonautonomous complex wave solutions for the $(2+1)$-dimensional nonlinear Schrödinger equation with variable coefficients in nonlinear optical fibers. Optik 180, 917-923 (2019)

8. Agarwal, P., Baltaeva, U., Alikulov, Y.: Solvability of the boundary-value problem for a linear loaded integro-differential equation in an infinite three-dimensional domain. Chaos Solitons Fractals 140, 110108 (2020)

9. Osman, M.S., Rezazadeh, H., Eslami, M.: Traveling wave solutions for $(3+1)$ dimensional conformable fractional Zakharov-Kuznetsov equation with power law nonlinearity. Nonlinear Eng. 8(1), 559-567 (2019)

10. Valliammal, N., Ravichandran, C., Nisar, K.S.: Solutions to fractional neutral delay differential nonlocal systems. Chaos Solitons Fractals 138, 109912 (2020)

11. Ding, Y., Osman, M.S., Wazwaz, A.M.: Abundant complex wave solutions for the nonautonomous Fokas-Lenells equation in presence of perturbation terms. Optik 181, 503-513 (2019)

12. Agarwal, P., Akbar, M., Nawaz, R., Jleli, M.: Solutions of system of Volterra integro-differential equations using optimal homotopy asymptotic method. Math. Methods Appl. Sci. (2020). https://doi.org/10.1002/mma.6783

13. Restrepo, J.E., Higuita, R.A., Jain, S.: Hyers-Ulam-Rassias stabilities of some classes of fractional differential equations. In: Special Functions and Analysis of Differential Equations, vol. 87 (2020)

14. Osman, M.S., Rezazadeh, H., Eslami, M., Neirameh, A., Mirzazadeh, M.: Analytical study of solitons to Benjamin-Bona-Mahony-Peregrine equation with power law nonlinearity by using three methods. UPB Sci. Bull., Ser. A 80(4), 267-278 (2018)

15. Ali, K.K., Wazwaz, A.M., Osman, M.S.: Optical soliton solutions to the generalized nonautonomous nonlinear Schrödinger equations in optical fibers via the sine-Gordon expansion method. Optik 208, 164132 (2020)

16. Jain, S., Nieto, J.J., Singh, G., Certain, C.J.: Generating relations involving the generalized multi-index Bessel-Maitland function. Math. Probl. Eng. 2020, Article ID 8596736 (2020) 
17. Menaceur, A., Boulaaras, S., Alkhalaf, S., Jain, S.: Limit cycles of a class of polynomial differential systems bifurcating from the periodic orbits of a linear center. Symmetry 12(8), 1346 (2020)

18. Shimano, R., Tsuji, N.: Higgs mode in superconductors. Annu. Rev. Condens. Matter Phys. 11, 103-124 (2020)

19. Yablonovitch, E.: Inhibited spontaneous emission in solid-state physics and electronics. Phys. Rev. Lett. 58(20), 2059 (1987)

20. Osman, M.S., Baleanu, D., Adem, A.R., Hosseini, K., Mirzazadeh, M., Eslami, M.: Double-wave solutions and Lie symmetry analysis to the $(2+1)$-dimensional coupled Burgers equations. Chin. J. Phys. 63, 122-129 (2020)

21. Osman, M.S., Inc, M., Liu, J.G., Hosseini, K., Yusuf, A.: Different wave structures and stability analysis for the generalized $(2+1)$-dimensional Camassa-Holm-Kadomtsev-Petviashvili equation. Phys. Scr. 95(3), 035229 (2020)

22. El-Sherif, A.A.: Mixed ligand complex formation reactions and equilibrium studies of Cu (II) with bidentate heterocyclic alcohol (N, O) and some bio-relevant ligands. J. Solution Chem. 39(1), 131-150 (2010)

23. Tian, B., Shan, W.R., Zhang, C.Y., Wei, G.M., Gao, Y.T.: Transformations for a generalized variable-coefficient nonlinea Schrödinger model from plasma physics, arterial mechanics and optical fibers with symbolic computation. Eur. Phys. J. B 47(3), 329-332 (2005)

24. Ali, K.K., Osman, M.S., Abdel-Aty, M.: New optical solitary wave solutions of Fokas-Lenells equation in optical fiber via sine-Gordon expansion method. Alex. Eng. J. 59,1191-1196 (2020)

25. Jarmolinski, A., Dobrowolski, T.: The role of magnetic fields for curvature effects in Josephson junction. Physica B 514, 24-29 (2017)

26. Lermusiaux, P.F.: Uncertainty estimation and prediction for interdisciplinary ocean dynamics. J. Comput. Phys. 217(1), 176-199 (2006)

27. Abdel-Gawad, H.I., Osman, M.: Exact solutions of the Korteweg-de Vries equation with space and time dependent coefficients by the extended unified method. Indian J. Pure Appl. Math. 45(1), 1-12 (2014)

28. Ali, K.K., Cattani, C., Gómez-Aguilar, J.F., Baleanu, D., Osman, M.S.: Analytical and numerical study of the DNA dynamics arising in oscillator-chain of Peyrard-Bishop model. Chaos Solitons Fractals 139, 110089 (2020)

29. Osman, M.S., Baleanu, D., Tariq, K.U.H., Kaplan, M., Younis, M., Rizvi, S.T.R.: Different types of progressive wave solutions via the 2D-chiral nonlinear Schrödinger equation. Front. Phys. 8, 215 (2020)

30. Hosseini, K., Ma, W.X., Ansari, R., Mirzazadeh, M., Pouyanmehr, R., Samadani, F.: Evolutionary behavior of rational wave solutions to the (4 + 1)-dimensional Boiti-Leon-Manna-Pempinelli equation. Phys. Scr. 95(6), 065208 (2020)

31. Tahir, M., Kumar, S., Rehman, H., Ramzan, M., Hasan, A., Osman, M.S.: Exact traveling wave solutions of Chaffee-Infante equation in (2+1)-dimensions and dimensionless Zakharov equation. Math. Methods Appl. Sci. (2020). https://doi.org/10.1002/mma.6847

32. Olver, P.J.: Hamiltonian and non-Hamiltonian models for water waves. Lect. Notes Phys. 195, 273-290 (1984)

33. Sawada, K., Kotera, T.: A method for finding N-soliton solutions of the KdV equation and the KdV-like equation. Prog. Theor. Phys. 51, 1355-1367 (1974)

34. Adomian, G.: The fifth-order Korteweg-de Vries equation. Int. J. Math. Math. Sci. 19(2), 415 (1996)

35. Nuruddeen, R.I.: Multiple soliton solutions for the $(3+1)$ conformable space-time fractional modified Korteweg-de Vries equations. J. Ocean Eng. Sci. 3, 11-18 (2018)

36. Djidjeli, K., Price, W.G., Twizell, E.H., Wang, Y.: Numerical methods for the soltution of the third and fifth-order disprsive Korteweg-de Vries equations. J. Comput. Appl. Math. 58, 307-336 (1995)

37. Kaya, D.: An application for the higher order modified KdV equation by decomposition method. Commun. Nonlinear Sci. Numer. Simul. 10, 693-702 (2005)

38. Wazwaz, A.M.: Multiple soliton solutions and other exact solutions for a two-mode KdV equation. Math. Methods Appl. Sci. 40(6), 2277-2283 (2017)

39. Gokdogan, A., Yildirim, A., Merdan, M.: Solving coupled-KdV equations by differential transformation method. World Appl. Sci. J. 19, 1823-1828 (2012)

40. Lei, Y., Fajiang, Z., Yinghai, W.: The homogeneous balance method, Lax pair Hirota transformation and a general fifth order KdV equation. Chaos Solitons Fractals 13(2), 337-340 (2002)

41. Seadawy, A.R., Nuruddeen, R.I., Aboodh, K.S., Zakariya, Y.F.: On the exponential solutions to three extracts from extended fifth-order KdV equation. J. King Saud Univ., Sci. 32, 765-769 (2020)

42. El-Wakil, S.A., Abulwafa, E.M., Zahran, M.A., Mahmoud, A.A.: Time-fractional KdV equation: formulation and solution using variational methods. Nonlinear Dyn. 65, 55-63 (2011)

43. Pandir, Y., Gurefe, Y., Misirli, E.: New exact solutions of the time fractional nonlinear dispersive KdV equation. Int. J. Model. Optim. 3(4), 349 (2013)

44. Islam, M.T., Akbar, M.A., Azad, M.A.K.: A rational (G/G)-expansion method and its application to modified KdV-Burgers equation and the (2+1)-dimensional Boussineq equation. Nonlinear Stud. 22(4), 635-645 (2015)

45. Yun-hu, W., Yong, C.: The integrability of an extended fifth-order KdV equation with Riccati-type pseudopotential. Pramana 81, 737-746 (2013)

46. Saad, M., Elagan, S.K., Hamed, Y.S., Sayed, M.: Using a complex transformation to get an exact solutions for fractional generalized coupled MKDV and KDV equations. Int. J. Basic Appl. Sci. 13, 23-25 (2013)

47. Bakodah, H.O.: Modified Adomian decomposition method for the generalized fifth order KdV equations. Am. J. Comput. Math. 3, 53-58 (2013)

48. Khalil, R., Al-Horani, M., Yousef, A., Sababheh, M.: A new definition of fractional derivative. J. Comput. Appl. Math. 265, 65-701 (2014)

49. Khalid, K.A., Nuruddeen, R.I., Raslan, K.R.: New hyperbolic structures for the conformable time-fractional variant bussinesq equations. Opt. Quantum Electron. 50(2), 61 (2018)

50. Kumar, A., Chauhan, H.V.S., Ravichandran, C., Nisar, K.S., Baleanu, D.: Existence of solutions of non-autonomous fractional differential equations with integral impulse condition. Adv. Differ. Equ. 2020, 434 (2020)

51. Alqudah, M.A., Ravichandran, C., Abdeljawad, T., Valliammal, N.: New results on Caputo fractional-order neutral differential inclusions without compactness. Adv. Differ. Equ. 2019, 528 (2019)

52. Seadawy, A.R., Khalid, K.A., Nuruddeen, R.I.: A variety of soliton solutions for the fractional Wazwaz-Benjamin-Bona-Mahony equations. Results Phys. 12, 2234-2241 (2019)

53. Khalid, K.A., Nuruddeen, R.I., Raslan, K.R.: New structures for the space-time fractional simplified MCH and SRLW equations. Chaos Solitons Fractals 106, 304-309 (2018) 
54. Ravichandran, C., Logeswari, K., Panda, S.K., Nisar, K.S.: On new approach of fractional derivative by Mittag-Leffler kernel to neutral integro-differential systems with impulsive conditions. Chaos Solitons Fractals 139, 110012 (2020)

55. Jothimani, K., Valliammal, N., Ravichandran, C.: Existence result for a neutral fractional integro-differential equation with state dependent delay. J. Appl. Nonlinear Dyn. 7(4), 371-381 (2018)

56. Osman, M.S., Lu, D., Khater, M.M.A., Attia, R.A.M.: Complex wave structures for abundant solutions related to the complex Ginzburg-Landau model. Optik 192, 162927 (2019)

57. Liu, J.G., Osman, M.S., Zhu, W.H., Zhou, L., Ai, G.P.: Different complex wave structures described by the Hirota equation with variable coefficients in inhomogeneous optical fibers. Appl. Phys. B 125(9), 175 (2019)

58. Abdel-Gawad, H.I., Osman, M.S.: On the variational approach for analyzing the stability of solutions of evolution equations. Kyungpook Math. J. 53(4), 661-680 (2013)

59. Nuruddeen, R.I., Aboodh, K.S., Ali, K.K.: Investigating the tangent dispersive solitary wave solutions to the equal width and regularized long wave equations. J. King Saud Univ., Sci. 32, 677-681 (2020)

60. Javid, A., Raza, N., Osman, M.S.: Multi-solitons of thermophoretic motion equation depicting the wrinkle propagation in substrate-supported graphene sheets. Commun. Theor. Phys. 71(4), 362 (2019)

61. Raslan, K.R., Talaat, S.E., Khalid, K.A.: Exact solution of space-time fractional coupled EW and coupled MEW equations Eur. Phys. J. Plus 132, 319 (2017)

\section{Submit your manuscript to a SpringerOpen ${ }^{\circ}$ journal and benefit from:}

- Convenient online submission

Rigorous peer review

- Open access: articles freely available online

- High visibility within the field

- Retaining the copyright to your article

Submit your next manuscript at $\boldsymbol{~ s p r i n g e r o p e n . c o m ~}$ 